\title{
ON THE SMOOTH STRUCTURES ON ELLIPTIC SURFACES AND RELATED TOPICS
}

\author{
By Masaaki Ue
}

A compact complex surface is called an elliptic surface if it admits a holomorphic projection to a Riemann surface whose general fiber (the inverse image of a regular value of the projection) is a nonsingular elliptic curve. Some of the total spaces of the elliptic surfaces as closed topological 4-manifolds have infinitely many smooth structures. Therefore they provide typical examples of exotic phenomena which occur only in dimension 4. In particular to treat the homeomorphism types and the diffeomorphism types of the elliptic surfaces when their fundamental groups are finite cyclic we need the deep results originated from Freedman and Donaldson. Whereas in case of those whose fundamental groups are not finite cyclic, they can be treated in more elementary way although in general we have no theories applicable to all 4-manifolds with given fundamental groups. Moreover there are still very few 4-manifolds other than the elliptic surfaces whose Donaldson invariants are explicitely computed (although they are not completely known even in the elliptic surfaces). So in this note we will describe some of such topics. In $\S 1$ we collect some known facts about the homeomorphism types and the diffeomorphism types of the elliptic surfaces. In $\S 2$ we describe the smooth structures on some elliptic surfaces not coming from complex surfaces and "exotic" free actions on some 4-manifolds derived from them ([U3], [U4]). The details of their proofs will appear elsewhere.

\section{$\S 1$. Some known facts about elliptic surfaces}

Let $\pi: S \rightarrow B$ be a compact elliptic surface over an Riemann surface $B$. Here we can assume without loss of generality that $S$ is relatively minimal. This means that $S$ has no (-1)-curve in any fiber. This is because if $S$ is not relatively minimal then the finitely many blowing downs provide a relatively minimal one. So we assume this for the rest of this note. Furthermore we will concentrate on the topology of the total space $S$ of the elliptic surface and so we ignore its complex structure itself (in particular any diffeomorphic elliptic surfaces are identified in this note). For more comprehensive investigation about the topology of them including their deformation types, see the book of Friedman and Morgan ([FM4]).

Let $e(S)$ be the euler number of $S$. Then it is known (by Noether formula) that $e(S) \geqq 0$ and it must be a multiple of 12 . Then $S$ belongs to one of the following classes.

(I) $e(S)=0$

An elliptic surface belongs to this class if and only if it contains only multiple tori (defined below) as its singular fibers. The elliptic surfaces in this case were completely

Received June 12, 1993. 
classified. Any two homeomorphic elliptic surfaces in this class are actually diffeomorphic. In particular the diffeomorphism types of all examples in this class are determined by their fundamental groups except for the elliptic ruled surfaces and the Hopf surfaces with abelian fundamental groups. The last exceptions are diffeomorphic to the direct products of (any) 3-dimensional lens spaces and the circle. Any two of such surfaces are diffeomorphic if and only if the corresponding lens spaces are diffeomorphic.

(II) $e(S)>0$

This case is divided into two subclasses.

(1) the fundamental group $\pi_{1} S$ is not finite cyclic.

(2) $\pi_{1} S$ is finite cyclic.

In either case $S$ contains some singular fibers other than multiple tori. $S$ belongs to (II-2) if and only if the base $B$ of $S$ has genus 0 and the number of multiple tori is at most 2. Moreover the diffeomorphism type of $S$ in case (II-1) is uniquely determined by its euler number $e(S)$ and the fundamental group $\pi_{1} S([\mathrm{M}],[\mathrm{Mt}]$ for special cases, and [U1] for general cases). In particular the topological classification of the elliptic surfaces is the same as the differentiable one if they do not belong to case (II-2). On the other hand the situation for the elliptic surfaces in case (II-2) is completely different. To treat them we prepare some notations.

Definition 1-1. A singular fiber $F$ of the elliptic surface $\pi: S \rightarrow B$ (the inverse image of the critical value of $\pi$ ) is called a multiple torus of multiplicity $m$ (denoted by ${ }_{m} I_{0}$ in Kodaira's notation [K]) if $F$ itself is a nonsingular elliptic curve but locally a general fiber $f$ covers $m$ times on $F$ (as a divisor $m F=f$ ).

The tubular neighborhood of the multiple torus $F=F_{m}$ of multiplicity $m$ is the inverse image by $\pi$ of the disk neighborhood $D$ of $\pi\left(F_{m}\right)$ in $B$ and is diffeomorphic to $D^{2} \times T^{2}$. But topologically the projection $\pi$ restricted to $\pi^{-1}(D)$ looks like:

$$
\pi:\left(D^{2} \times T^{2}\right) / \mathbf{Z}_{m} \rightarrow D^{2} / \mathbf{Z}_{m}
$$

where the generator $\rho$ of the cyclic group $\mathbf{Z}_{m}$ of order $m$ acts on the 2-disk $D^{2}$ by the rotation through angle $2 \pi / m$ centered at the origin 0 and acts on $D^{2} \times T^{2}$ by

$$
\rho(z, \theta, \varphi)=(\exp (2 \pi i / m) z, \theta-p / m, \varphi-q / m)
$$

for $(z, \theta, \varphi) \in D^{2} \times T^{2}$ with $z \in \mathbf{C},|z|=1, \theta, \varphi \in \mathbf{R} \quad(\bmod \mathbf{Z})$ such that $p$ and $q$ are some integers satisfying $\operatorname{gcd}(m, p, q)=1$. The projection $\pi$ is induced from the projection from $D^{2} \times T^{2}$ to the first factor and the multiple torus $F_{m}$ coincides with $\left(\{0\} \times T^{2}\right) / \mathbf{Z}_{m}$. We call $F_{m}$ in the above representation a multiple torus of type $(m, p, q)$ for the moment.

There are several singular fibers other than multiple tori. Their types were classified by Kodaira ([K], [BVP]). In particular there are multiple fibers with multiplicity $m$ other than multiple tori denoted by ${ }_{m} I_{b}(b \geqq 1)$ in Kodaira's notation. But by deformation of the complex structures of the total space they are reduced by the sum of one multiple torus with the same multiplicity and several non-multiple singular fibers $([\mathrm{M}])$. So we have only to consider the elliptic surfaces whose multiple fibers are multiple tori only. Moreover it is known that the diffeomorphism type of $S$ is completely determined once we fix the genus $g$ of the base space $B$, the euler number $e(S)=12 k$ (say) of $S$, and the set of multiplicities $\left(m_{1}, \ldots, m_{s}\right)$ of the multiple tori contained in $S$ if $e(S)>0$ or equivalently $k \geqq 1$ ([U1]). (Note that $k=\chi(\mathcal{O})=1-q+p_{g}$ where $\mathcal{O}$ is the structure 
sheaf of $S, q=\operatorname{dim} H^{1}(S, \mathcal{O})$, and $p_{g}=\operatorname{dim} H^{2}(S, \mathcal{O})$ by Noether formula.) The above fact comes from the fact that we can assume that the types of the multiple tori are $\left(m_{i}, 1,0\right)(i=1, \ldots, s)$ at the same time. This is proved by the existence of the nonmultiple singular fibers in $S$ (in particular the non-trivial local monodromies around such fibers). We also note that the above claim is not true if $e(S)=0$. Furthermore we note that the elliptic surface with such a multiple torus with multiplcity $m$ is produced from that without multiple fibers by logarithmic transform of multiplicity $m$.

Definition 1-2. Let $\pi: S \rightarrow B$ be an elliptic surface, $f$ be its general fiber, and $N(f)$ be the tubular neighborhood of $f . N(f)$ is identified with $T^{2} \times D^{2}$ so that $S^{1} \times S^{1} \times *$ for any one point $* \in \partial D^{2}$ is parallel to $f\left(* \times * \times \partial D^{2}\right.$ corresponds to a cross section $Q$ ). Put $L=* \times S^{1} \times *$ and $H=S^{1} \times * \times *$. Now for any $m \geqq 2, p, q$ with $\operatorname{gcd}(m, p, q)=1$ we can define a 4 -manifold $S^{\prime}$ obtained from $S$ by removing $N(f)$ from $S$ and attaching $T^{2} \times D^{2}$ by some diffeomorphism $\varphi: T^{2} \times \partial D^{2} \rightarrow \partial(S-N(f))$ such that $\varphi\left(* \times \partial D^{2}\right)$ is homologous to $m Q+p L+q H$. Then $S^{\prime}$ is again diffeomorphic to an elliptic surface with projection $\pi^{\prime}$ to the same base space $B$ such that the core of the new $T^{2} \times D^{2}$ is the multiple torus $F_{m}$ of multiplicity $m$ (of type $(m, p, q)$ ). In this case $S^{\prime}$ is called an elliptic surface obtained from $S$ by logarithmic transform of multiplicity $m$ at $f$.

See $[\mathrm{K}]$, [BPV] for the details. Topologically the logarithmic transform is the surgery along the 2-torus $f$. Note that this operation makes sense even if $m=1$ (in this case $S^{\prime}$ is the same as the original $S$ ) and even if $m=0$. In the last case the resulting manifold $S^{\prime}$ is no longer a complex surface but is defined as a smooth 4-manifold. We can see that the effect of this surgery depends only on the multiplicity $m$ (even if $m=0$ ) if $e(S)>0$, i.e., the diffeomorphism type of $S^{\prime}$ depends only on $m$ (not depending on $p$ and $q$ ) if $e(S)=e\left(S^{\prime}\right)>0$. Since from now on we call particular attention to the cases when the base spaces have genus 0 , we will denote the elliptic surface of the above type with $g=0$ by $S_{k}\left(m_{1}, \ldots, m_{s}\right)$. For convenience we assume that the multiple torus of multiplicity 1 means the general fiber and that $S_{k}\left(1, m_{2}, \ldots, m_{s}\right)=S_{k}\left(m_{2}, \ldots, m_{s}\right)$.

If the elliptic surface $S$ has multiple fibers then its base space $B$ can be considered as a 2-orbifold whose singular points are exactly the images of the multiple fibers by the projection which have the same multiplicities as those of the corresponding fibers. Then if $e(S)>0$ the fundamental group $\pi_{1} S$ of $S$ coincides with the orbifold fundamental group $\pi_{1}^{\text {orb }} B$ of $B$ as the 2-orbifold (cf. [U1]). it is easy to see that the type of the 2-orbifold $B$ is uniquely determined by $\pi_{1} S$ unless $\pi_{1} S$ is finite cyclic. $S$ belongs to the exceptions just when $S=S_{k}\left(m_{1}, m_{2}\right)$ in which case $\pi_{1} S$ is cyclic of order $\operatorname{gcd}\left(m_{1}, m_{2}\right)$. These cases are the only examples in the elliptic surfaces in which the topological classification and the differentiable one are not the same. The topological classification of them comes from Freedman's theorem in 1-connected cases and from Hambleton-Kreck's results ([HK1-3]) in general cases as follows.

Proposition 1-3. $S_{k}(p, q)$ and $S_{k}^{\prime}\left(p^{\prime}, q^{\prime}\right)$ are homeomorphic if and only if $k=k^{\prime}$, $\operatorname{gcd}(p, q)=\operatorname{gcd}\left(p^{\prime}, q^{\prime}\right)($ we denote it by $r)$ and

(1) both $r$ and $k$ are odd, or

(2) otherwise $(p+q) / r \equiv\left(p^{\prime}+q^{\prime}\right) / r \quad(\bmod 2)$. 
Hambleton-Kreck ([HK1-3]) proved that the homeomorphism types of closed oriented 4-manifolds with finite cyclic fundamental groups are classified by their intersection forms on the second homology groups with coefficients $\mathbf{Z}$ (modulo torsion), their Kirby-Siebenmann invariants, and their $w_{2}$-types (the informations on the second StiefelWhitney classes of them and their universal coverings). This was proved in [HK1] for the cases with odd cyclic fundamental groups. In case with even cyclic fundamental groups this was proved in [HK2] for some restricted cases and in [HK3] for the general cases. But we note that to prove the above proposition the results for the restricted cases in $[\mathrm{HK} 1,2]$ are sufficient.

To distiguish the diffeomorphism types of $S_{k}(p, q)$ 's we need the deep results about Donaldson's invariants. In fact Friedman-Morgan, Okonek -Van de Ven and others proved that many elliptic surfaces of these types have infinitely many smooth structures within the class of elliptic surfaces ([FM1-4], [OV]). In particular

ThEOREM 1-4 [FM1-4]. (1) $S_{1}(p, q)$ with $\operatorname{gcd}(p, q)=1(p, q>1)$ has infinitely many smooth structures coming from the other elliptic surfaces. In partıcular $S_{1}(2, p)$ is diffeomorphic to $S_{1}(2, q)$ if and only if $p=q$.

(2) If $S_{k}(p, q)$ is diffeomorphic to $S_{k}\left(p^{\prime}, q^{\prime}\right)$ for $k \geqq 2$ and $\operatorname{gcd}(p, q)=\operatorname{gcd}\left(p^{\prime}, q^{\prime}\right)=1$, or $\operatorname{gcd}(p, q)=\operatorname{gcd}\left(p^{\prime}, q^{\prime}\right)>1$ then $p q=p^{\prime} q^{\prime}$.

The claim (1) was proved by computation of Donaldson's $\Gamma$ invariants [FM1] (see also [B]) and (2) was proved by Donaldson's $S U(2)$ polynomial invariants ([FM2,4]). Both were based on the algebro-geometric methods coming from the computation of the moduli spaces of certain stable vector bundles over the elliptic surfaces. The proof for non-simply connected cases (the cases with $\operatorname{gcd}(p, q) \neq 1$ ) is deduced from that for 1connected cases since the universal covering of $S_{k}(p, q)$ for $\operatorname{gcd}(p, q)=r$ is $S_{k r}(p / r, q / r)$. It is very likely that the following conjecture is true.

ConjeCture. $S_{k}(p, q)$ and $S_{k}\left(p^{\prime}, q^{\prime}\right)$ with $p \geqq q \geqq 1$ and $p^{\prime} \geqq q^{\prime} \geqq 1$ are diffeomorphic if and only if $p=p^{\prime}$ and $q=q^{\prime}$ except for the following cases.

\section{(*) $S_{1}(1, q)$ is diffeomorphıc to $\mathbf{C P}^{2} \sharp 9 \overline{\mathbf{C P}} \mathbf{P}^{2}$ for any $q \geqq 1$}

The last exceptions are all rational elliptic surfaces as is well-known by Castelnuovo's criterion. More recently the above conjecture was solved affirmatively when $R \geqq 2$ by [MM].

Finally we also note that 1-connected elliptic surfaces have decompositions by Brieskorn homology 3-spheres corresponding to some orthogonal decompositions of their intersection forms ([G], [U3]). In particular $S_{k}(p, q)$ contains a compact submanifold $N_{k}(p, q)$ with second betti number 2 whose boundary is the Brieskorn homology sphere $\Sigma(2,3,6 k-1)$ with its orientation reversed and which contains all the multiple tori in $S_{k}(p, q)$. Such a submanifold which is called a nucleus by Gompf includes all the effect of logarithmic transforms. 
§. The smooth structures on the elliptic surfaces not coming from complex surfaces

Now it is also known that some elliptic surfaces are homeomorphic to 4-manifolds not diffeomorphic to any complex surfaces. Such examples for 1-connected cases are deduced directly from Freedman and Donaldson's works as follows.

(2-1) See [FQ],[DK]. A relatively minimal 1-connected elliptic surface $S$ (which is of the form $S_{k}(p, q)$ with $\left.k \geqq 1, \operatorname{gcd}(p, q)=1\right)$ is homeomorphic to $(k / 2) K 3 \sharp(k / 2-1) S^{2} \times$ $S^{2}$ if $k$ is even and both $p$ and $q$ are odd, and is homeomorphic to $(2 k-1) \mathbf{C P}^{2} \sharp(10 k-$ 1) $\overline{\mathbf{C P}^{2}}$ otherwise. Here $K 3$ means the K3 surface (note that the diffeomorphism type of the K3 surfaces is unique). The latter one in either case is not diffeomorphic to a complex surface except for $S_{1}(1,1)=\mathbf{C} P^{2} \sharp 9 \overline{\mathbf{C P}^{2}}$ and $S_{2}(1,1)=K 3$.

The last claim comes from Donaldson's theorem since any 1-connected complex surface is not diffeomorphic to a 4-manifold which is a connected sum of two indefinite 4-manifolds (the intersection forms are indefinite). The generalization of (2-1) to some non-simply connected cases were proved by Hambleton-Kreck [HK3].

(2-2) [HK3]. Let $S$ be an elliptic surface with finte fundamental group and with $k=e(S) / 12 \geqq 2$. Then there exist at least one smooth 4-manifold homeomorphic to $S$ whose universal covering is a connected sum of two indefinite 4-manifolds (which is not diffeomorphic to a complex surface by Donaldson's theorem). (If $k=1$ the same claim holds for $S \sharp \mathbf{C P}^{2}$ in place of $S$.)

Note that $S$ has finite fundamental group if and only if $S=S_{k}(p, q)$ with $p, q \geqq 1$ or $S=S_{k}(p, q, r)$ with $p, q, r \geqq 2$ and $1 / p+1 / q+1 / r>1$. In the second case $\pi_{1} S$ is a noncyclic polyhedral subgroup of $S O(3)$.

It seems difficult to obtain two or more examples which are connected sum of indefinite 4-manifolds which are homeomorphic to elliptic surfaces since all the Donaldson invariants for such decomposable 4-manifolds vanish in general and so we have no method to distinguish them. On the other hand for some elliptic surfaces there exist infinitely many indecomposable 4-manifolds homeomorphic to them but non-diffeomorphic to any complex surfaces. First such examples were discovered by Gompf-Mrowka [GM].

Definition 2-3. A closed oriented smooth 4-manifold $X$ is called irreducible if $X$ can be diffeomorphic to an connected sum of two 4-manifolds $M \sharp N$ only when either $M$ or $N$ is a homotopy 4-sphere (and hence homeomorphic to the 4-sphere by Freedman's theorem).

At present we have no way to see whether homotopy 4-spheres are diffeomorphic to the real 4-sphere and hence the above definition is considered modulo homotopy 4-spheres.

(2-4) [GM]. For any 1-connected elliptıc surface $S$ of the form $S=S_{2}(p, q)$ with $\operatorname{gcd}(p, q)=1$ (ıncluding the K3 surface) there exist infinitely many mutually non- 
diffeomorphic smooth 4-manifolds homeomorphic to $S$ such that any of them is irreducible but never diffeomorphic to a complex surface.

Actually their examples were the first counterexamples to Thom's conjecture ([FM3]) which asserts that any closed smooth 1-connected irreducible 4-manifold is diffeomorphic to a complex surface or the real 4-sphere. We can extend their results to those for some elliptic surfaces with larger euler numbers using their methods as follows.

THEOREM 2-5 [U3-4]. Let $X$ be a relatıvely minimal elliptic surface with $k=$ $e(X) / 12$ and with $\pi_{1} X$ finite. If $\pi_{1} X$ is cyclic of order $r$, then assume further that $r k \not \equiv 0 \quad$ (mod 4) and also that the multiplicites of the multıple fibers divided by $r$ are all odd if $r k \equiv 2 \quad(\bmod 4)$. Then there exist infinitely many smooth 4-manifolds $X_{2}$ $(i=1, \ldots)$ homeomorphic to $X$ such that the unvversal covering $\tilde{X}_{8}$ of $X_{2}$ are mutually non-diffeomorhic and irreducible and none of $X_{\imath}$ is diffeomorphic to a complex surface (or a complex surface with its orientation reversed).

Corollary 2-6. For any given $N>0$ there exists a closed smooth 1-connected ırreducible 4-manifold non-diffeomorphic to a complex surface such that its Euler number is greater than $N$.

Now in [FS] Fintushel and Stern gave many examples of 1-connected irreducible 4manifolds homeomorphic to elliptic surfaces and algebraic surfaces of general type which have infinitely many smooth structures.

CoRollary 2-7. For any finite subgroup $\pi$ of $S O(3)$ there exist infinitely many closed smooth irreducible 4-manifolds with fundamental group isomorphic to $\pi$ each of which has infinitely many smooth structures.

This also comes directly from Theorem 2-5 since any such $\pi$ can be realized by the fundamental group of some elliptic surfaces appeared in Theorem 2-5. The examples in Theorem 2-5 are constructed as follows. We start with $X=S_{k}(p, q, r)$ with $1 / p+$ $1 / q+1 / r>1$ or $X=S_{k}(r, r)$ (if $\pi_{1} X$ is finite cyclic then the assumption in Theorem 2-5 and Hambleton-Kreck's theorem show that $X$ is homeomorphic to the second one). Let $S_{k}^{0}\left(m_{1}, \ldots, m_{s}\right)$ be the 4-manifold obtained from $S_{k}\left(m_{1}, \ldots, m_{s}\right)$ by removing the tubular neighborhood of the general fiber of $S_{k}\left(m_{1}, \ldots, m_{s}\right)(k \geqq 2)$. For the pairs of integers $\left(p_{i}, q_{i}\right)$ satisfying

$$
\text { (C) } \operatorname{gcd}\left(p_{i}, q_{i}\right)=1, \quad p_{i} \equiv q_{i} \equiv 1 \quad(\bmod 2)
$$

put $S_{i}^{\sigma}=S_{1}^{0}\left(p_{i}, q_{i}\right) \cup_{\sigma} S_{(k-1)}^{0}(p, q, r)$ or $S_{1}^{0}\left(p_{i}, q_{i}\right) \cup_{\sigma} S_{(k-1)}^{0}(r, r)$ where $\sigma$ is a diffeomorphism between the boundaries of the above two compact manifolds (each of them is a 3-torus) which does not preserve the general fibers contained in both pieces (we can choose any such $\sigma$ and fix it). It should be noted that for some technical reasons in case of $X=S_{2}(p, q)$ with $\operatorname{gcd}(p, q)=1$ (this is the case that Gompf-Mrowka considered originally) they needed more logarithmic transforms to construct the desired examples in [GM]. For all other cases the above constructions suffice. Under the condition (C) $S_{i}^{\sigma}$ is homeomorphic to $X$. This comes from the fact that $S_{i}^{\sigma}$ contains naturally a copy of the "nucleus" $N_{2}\left(p_{i}, q_{i}\right)$ which contains the multiple tori in $S_{1}^{0}\left(p_{i}, q_{i}\right)$ but disjoint from the 
other multiple fibers in $S_{i}^{\sigma}([\mathrm{GM}])$ and that there is a homeomorphism between $N_{2}\left(p_{i}, q_{i}\right)$ and $N_{2}(1,1)$ which is the identity on the boundaries ([G]). Moreover we also note that $S_{i}^{\sigma}$ is diffeomorphic to the original $X$ if $p_{i}=q_{i}=1$ since any self-diffeomorphism on the boundary of $S_{1}^{0}(1,1)$ extends to that of $S_{1}^{0}(1,1)$. So it should be noted that the assertion about the homeomorphisms for our cases depends only on Freedman's topological classification of 1-connected 4-manifolds even if the total space is not 1-connected (contrary to the results in [HK3]). To distinguish the diffeomorphism types of our examples we give some estimates of the "simple invariants" of their universal coverings. The simple invariants (Donaldson's $S O(3)$ invariants of degree 0 ) for a 1-connected 4-manifold $X$ with $b^{+}(X)=2 k-1>1$ (where $b^{+}(X)$ is the rank of the maximal positive subspace of the intersection form of $X$ ) are given by the integer-valued map $\gamma_{X}$ from the class $\mathcal{C}_{X}$ defined by

$$
\mathcal{C}_{X}=\left\{\eta \in H^{2}\left(X, \mathbf{Z}_{2}\right) \mid \eta \neq 0, \eta \cdot \eta \equiv \ell_{X} \quad(\bmod 4)\right\}
$$

where $\ell_{X}=-3\left(1+b^{+}(X)\right) / 2$. The value $\gamma_{X}(\eta)$ is defined as the number of instantons on the $S O(3)$ bundle over $X$ with $w_{2}=\eta, p_{1}=\ell_{X}$ counted with sign for a generic Riemann metric on $X$ (see [DK], [GM] for the details). By the naturality of this value with respect to the oriented diffeomorphisms the maximum $\gamma_{X}^{\max }$ of $\left|\gamma_{X}(\eta)\right|$ for $\eta \in \mathcal{C}_{X}$ is the diffeomorphism invariant of $X$. For the estimates of the simple invariants of our examples we need the computation of them for the elliptic surfaces without multiple fibers ([DK], $[\mathrm{KS}]$ ) obtained by algebro-geometric method, the formula on the effect of logarithmic transforms due to Gompf-Mrowka [GM], and its generalization (product formula) by Kametani [Ka] together with the action of the group of self-diffeomorphisms of $X$ on $\mathcal{C}_{X}$ when $X$ is an elliptic surface (this is purely topological) [U3,4]. These observations applied to the universal covering $\widetilde{S}_{i}^{\sigma}$ of $S_{i}^{\sigma}$ show that $\gamma_{\widetilde{S}_{i}^{\sigma}}^{\max }$ tends to $\infty$ if $p_{i} q_{i}$ tends to $\infty$ and hence we can choose infinitely many mutually non-diffeomorphic manifolds among them. The irreducibility of them is deduced from further estimates of their simple invariants and connected sum formula due to Donaldson and Kotschick. Finally to show that our examples are not diffeomorphic to complex surfaces we need to consult Enriques-Kodaira's table on complex surfaces, Miyaoka-Yau's inequality on the Chern classes of the algebraic surfaces [BPV], and the comparison of the simple invariants of our examples with those for the elliptic surfaces ([U3,4]).

We can deform the constructions given in Theorem 1 to get infinitely many exotic 4manifolds whose universal coverings are all diffeomorphic. In fact let $L_{r}$ be a 4-manifold obtained from the direct product of the lens space $L(r, 1)$ and the circle by performing surgery along the circle $* \times S^{1}$. Then $L_{r}$ (whose diffeomorphism type does not depend on the choice of the surgery framing) is a rational homology 4 -sphere (and also a $\mathbf{Z}_{2}$ homology 4-sphere if $r$ is odd) with $\pi_{1} L_{r}=\mathbf{Z} / r \mathbf{Z}$. Then if $r$ is odd we can see that among the manifolds $X_{\imath}=L_{r} \sharp S_{i}^{\sigma}$ for $S_{i}^{\sigma}=S_{1}^{0}\left(p_{i}, q_{i}\right) \cup_{\sigma} S_{(k-1)}^{0}(1,1)$ there exist infinitely many mutually homeomorphic but non-diffeomorphic manifolds. The proof goes as in the previous cases since the simple invariants can be defined also when the fundamental group is odd cyclic ([U4]). Moreover the universal covering $\widetilde{X}_{i}$ of $X_{i}$ is diffeomorphic to a connected sum of $r-1$ copies of $S^{2} \times S^{2}$ (coming from the universal covering of $L_{r}$ ) and $r$ copies of $S_{i}^{\sigma}$ and to either a connected sum of some copies of $\pm \mathbf{C} P^{2}$ or a connected sum of copies of $K 3$ and $S^{2} \times S^{2}$. This comes from Mandelbaum's lemma ([Ma]) which was used to show that 1-connected elliptic surfaces are almost completely decomposable. 
Hence in this case the actions of the covering translations on $\widetilde{X}_{\imath}$ are "exotic". In other words we have

Theorem 2. Let $X=(2 n-1) \mathbf{C P}^{2} \sharp(10 n-1) \overline{\mathbf{C P}^{2}}$ or $X=n K 3 \sharp(n-1) S^{2} \times S^{2}$ where $n$ is divided by some odd integer $r$ with $1<r<n$. Then there exist infinitely many orientation-preserving free $\mathbf{Z}_{r}$ actions on $X$ such that their orbıt spaces are mutually homeomorphic but non-diffeomorphic to each other.

\section{REFERENCES}

[B] S. Bauer, Some nonreduced moduli of bundles and Donaldson invariants for Dolgachev surfaces, Jour. fur die reine und angew. Math. 424 (1992), 149-180.

[BPV] W. Barth, C. Peters, A, Van de Ven, Compact Complex Surfaces, Ergeb. der math., Springer, Berlin-New York, 1984.

[DK] S.K. Donaldson, P.B. Kronheimer, The Geometry of Four Manifolds, Oxford Math. Monographs, Oxford, 1990.

[FM1] R. Friedman, J. Morgan, On the differentiable clasfication of algebraic surfaces $I, I I, J$. Diff. Geom. 77 (1988), 297-369, 371-398.

[FM2] - Complex versus differentiable classification of algebraic surfaces, Topology and its appl. 32 (1989), 135-139.

[FM3] - Algebraic surfaces and 4-manifolds: some conjectures and speculations, Bull, Amer. Soc. 18 (1988), 1-19.

[FM4] - Smooth four-manifolds and complex surfaces, Springer, (1994).

[FQ] M. Freedman, F. Quinn, Topology of 4-manifolds, Princetion Math. Series 39, Princeton, 1990.

[FS] R. Fintushel, R. Stern, Surgery on cusp neighborhoods and the geography of irreducible 4manifolds, Invent. Math. 117 (1994), 455-524.

[G] R. Gompf, Nuclei of Elliptic surfaces, Topology 30 (1991), 479-512.

[GM] R. Gompf, T.S. Mrowka, Irreducible Four Manifolds need not be complex, in Ann. Math. 138 (1993), 61-111.

[HK1] I. Hambleton, M. Kreck, On the classification of topological 4- manifolds with finite fundamental group, Math. Ann. 280 (1988), 85-104.

[HK2] - Smooth structures on algebraic surfaces with cyclic fundamental group, Invent. Math. 91 (1988), 53-59.

[HK3] - Cancellation, elliptic surfaces and the topology of certain four manifolds, J. Reine Angew Math. 444 (1993), 79-100.

[K] K. Kodaira, On compact complex analytic surfaces I, Ann. of Math. 71 (1960), 111-152; II, Ann. of Math. 77 (1963), 563-626; III, Ann. of Math. 78 (1963), 1-40.

[Ka] Y. Kametani, A product formula of simple invariants on torus sum of 4-manifolds, Kodai Math. J. 16 (1993), 138-170.

[KS] Y. Kametani, Y. Sato, 0-dimensional moduli spaces of stable rank 2 bundles and differentiable structures on regular elliptic surfaces, Tokyo J. Math. (to appear).

[M] B. Moishezon, Complex Surfaces and Connected Sums of Complex Projective Planes, Lecture Notes in math. 603, Springer, 1977.

[Ma] R. Mandelbaum, Decomposing analytic surfaces, Geometric Topology, Academic Press, 1979, pp. 147-218.

[Mt] Y. Matsumoto, Diffeomorphism types of Elliptic Surfaces, Topology 25 (1986), 549-564.

[MM] J. Morgan, T.S. Mrowka, On the diffeomorphism classification of regular elliptic surfaces, Internat. Math. Res. Notices 6 (1993), 183-184. 
[OV] C. Okonek, A. Van de Ven, Stable vector bundles and differentiable structures on certain elliptic surfaces, Invent. Math 86 (1986), 357-370.

[U1] M. Ue, On the diffeomorhism types of elliptic surfaces with multiple fibers, Invent. Math. 84 (1986), 633-643.

[U2] - On the decompositions of elliptic surfaces, Knots 90: Proc. of the International Conference on Knot Thery and Related Topics, de Gruyter, 1992, pp. 299-322.

[U3] - Remarks on simple invariants for elliptic surfaces and their exotic structures not coming from complex surfaces, preprint (1992).

[U4] - A remark on the simple invariants for elliptic surfaces and the exotic free actions in dimension 4, preprint (1993).

Division OF MATHEMATICS

Faculty of Integrated Human Studies

KYOTO UNIVERSITY

606-01 KYOTO, JAPAN 